ESAIM: COCV 18 (2012) 889-913

DOI: $10.1051 / \mathrm{cocv} / 2011186$
ESAIM: Control, Optimisation and Calculus of Variations

www.esaim-cocv.org

\title{
ON SPECTRUM AND RIESZ BASIS PROPERTY FOR ONE-DIMENSIONAL WAVE EQUATION WITH BOLTZMANN DAMPING *
}

\author{
BaO-Zhu GuO ${ }^{1,2,4}$ And Guo-Dong Zhang ${ }^{2,3}$
}

\begin{abstract}
In this paper, we study the one-dimensional wave equation with Boltzmann damping. Two different Boltzmann integrals that represent the memory of materials are considered. The spectral properties for both cases are thoroughly analyzed. It is found that when the memory of system is counted from the infinity, the spectrum of system contains a left half complex plane, which is sharp contrast to the most results in elastic vibration systems that the vibrating dynamics can be considered from the vibration frequency point of view. This suggests us to investigate the system with memory counted from the vibrating starting moment. In the latter case, it is shown that the spectrum of system determines completely the dynamic behavior of the vibration: there is a sequence of generalized eigenfunctions of the system, which forms a Riesz basis for the state space. As the consequences, the spectrum-determined growth condition and exponential stability are concluded. The results of this paper expositorily demonstrate the proper modeling the elastic systems with Boltzmann damping.
\end{abstract}

Mathematics Subject Classification. 35L05, 47A10, 47E05, 58K55.

Received August 30, 2010

Published online September 27, 2011.

\section{INTRODUCTION}

The dynamics and control of vibration for elastic structures with or without viscoelasticity have attracted much attention over the past three decades, see for instance $[10,11,14,16,23,24]$ for beam equations, and $[13,17,21,25]$ for wave equations. A special property reported in $[10,11]$ for elastic systems says that even under the feedback control, the closed-loop system shares the same basis property as the free (uncontrolled) counterpart: there is a sequence of generalized eigenfunctions, which forms a Riesz basis for the state space. This shows that the dynamics of the vibrating system is determined completely by the vibration frequencies. Other studies from different aspects for elastic structures can also be found in $[1,3-5,7,8,15,18,20]$.

Among of these works, one of the most widely used models for viscoelasticity is the Boltzmann integral model, see $[2,17,21,23]$ and the references therein. This kind of passive control can now be accomplished as

Keywords and phrases. Wave equation, spectrum, Riesz basis, stability, Boltzmann damping.

* This work was supported by the National Natural Science Foundation of China, the National Basic Research Program of China with grant No. 2011CB808002, and the National Research Foundation of South Africa.

1 Academy of Mathematics and Systems Science, Academia Sinica, Beijing 100190, P.R. China. bzguo@iss.ac.cn

2 School of Computational and Applied Mathematics, University of the Witwatersrand, Wits 2050, Johannesburg, South Africa

3 School of Mathematical Science, Heilongjiang University, Harbin 150080, P.R. China

4 School of Mathematical Sciences, Shanxi University, Taiyuan 030006, P.R. China 
active vibration control through piezoelectric actuator/sensor ([22]). The Boltzmann type models attempt to capture the viscosity of the material and the history dependence of the stress on the strain and/or strain rate, which can be reduced easily to some well-known differential models, e.g., Kelvin-Voigt and Maxwell. Basically, there are two types of Boltzmann integrals. One is with the infinite entire memory $([2,15,17,18,23])$, and another is with finite memory $([3,8,21])$.

In this paper, we are interested in the difference between these two different types of Boltzmann integrals for the dynamics of vibrating systems. We use the one-dimensional wave equation with Boltzmann model of the viscoelasticity for expository demonstration. It is assumed that the instantaneous stress depends on the instantaneous strain and history of strain rate linearly. When the history is entire, that is, the memory is counted from $-\infty$ to $t$, then the stress $\sigma$ at time $t$ and position $x$ is ([17]):

$$
\begin{aligned}
\sigma(x, t) & =\int_{-\infty}^{t} \eta(x, t-s) \varepsilon_{t}(x, s) \mathrm{d} s \quad(\varepsilon(x,-\infty)=0) \\
& =\eta(x, \infty) \varepsilon(x, t)-\int_{0}^{\infty} \eta_{s}(x, s)[\varepsilon(x, t)-\varepsilon(x, t-s)] \mathrm{d} s \\
& =a(x) \varepsilon(x, t)-b(x) \int_{0}^{\infty} g_{s}(s)[\varepsilon(x, t)-\varepsilon(x, t-s)] \mathrm{d} s,
\end{aligned}
$$

while the memory is finite, that is, the memory is counted from the vibration starting moment 0 to $t$, the stress is:

$$
\begin{aligned}
\sigma(x, t) & =\int_{0}^{t} \eta(x, t-s) \varepsilon_{t}(x, s) \mathrm{d} s \quad(\varepsilon(x, 0)=0) \\
& =\eta(x, 0) \varepsilon(x, t)+\int_{0}^{t} \eta_{t}(x, t-s) \varepsilon(x, s) \mathrm{d} s \\
& =[a(x)+b(x) g(0)] \varepsilon(x, t)+\int_{0}^{t} b(x) g_{t}(t-s) \varepsilon(x, s) \mathrm{d} s
\end{aligned}
$$

where we take the relaxation function in the form of ([17])

$$
\eta(x, s)=a(x)+b(x) g(s), \quad g(\infty)=0 .
$$

So, the corresponding governing equation to infinite memory is ([17]):

$$
\left\{\begin{array}{l}
u_{t t}(x, t)=\left(a(x) u_{x}(x, t)-b(x) \int_{0}^{\infty} g_{s}(s)\left[u_{x}(x, t)-u_{x}(x, t-s)\right] \mathrm{d} s\right)_{x}, \\
u(0, t)=u(1, t)=0, \quad t>0,0<x<1, \\
u(x, t)=u_{0}(x, t), u_{t}(x, t)=u_{1}(x, t), \quad t \leq 0,0<x<1
\end{array}\right.
$$

and the equation to finite memory is ([21]):

$$
\left\{\begin{array}{l}
u_{t t}(x, t)=\left(a(x) u_{x}(x, t)+b(x) \int_{0}^{t} g_{t}(t-s) u_{x}(x, s) \mathrm{d} s\right)_{x}, x \in(0,1), t>0 \\
u(0, t)=u(1, t)=0 \\
u(x, 0)=u_{0}(x), u_{t}(x, 0)=u_{1}(x)
\end{array}\right.
$$

where in (1.5), we replace $a(x)+b(x) g(0)$ by $a(x)$ for the sake of simplicity. Hereafter, we use prime " " to represent the derivative with respect to $x$. 
In order to compare the models (1.4) and (1.5) qualitatively, we take the kernel simply as the finite sum of exponential polynomials, and both $a$ and $b$ are positive constant functions:

$$
\left\{\begin{array}{l}
g(s)=\sum_{j=1}^{N} a_{j} \mathrm{e}^{-b_{j} s}, 0<a_{j}, b_{j} \in \mathbb{R}, 1 \leq N \in \mathbb{N}, \\
a(x) \equiv a>0, b(x) \equiv b>0,
\end{array}\right.
$$

where it is assumed that

$$
0<b_{1}<b_{2}<\cdots<b_{N}
$$

It is noted that since we replace $a+b g(0)$ by $a$ in (1.5) and $a>0$ in modeling (1.2), it is natural to assume in (1.6) that

$$
a-b g(0)=a-b \sum_{j=1}^{N} a_{j}>0 .
$$

The system (1.4) has been formulated into an abstract evolution equation in [17] based on the idea of [6]. In next section, Section 2, the spectral analysis for this system with kernel (1.6) is thoroughly performed. The asymptotic distribution of eigenvalues is presented. It is shown that the spectrum of the system operator contains a half complex plane, which is an unexpected result for an elastic vibrating system.

Section 3 is devoted to the analysis of system (1.5), (1.6). We adapt the methods used in [23] for the heat equation with finite memory. The spectral analysis for the system operator that is not of resolvent compact shows that there is a sequence of generalized eigenfunctions of the system operator, which forms a Riesz basis for the state space. This is sharp contrast with the heat equation with memory discussed in [23], but coincides, in reflecting the dynamic behavior of system via the vibrating frequencies, with those presented in $[10,11]$ where the system operators are of compact resolvent. As the consequences, the spectrum-determined growth condition as well as the exponential stability of the system is concluded.

\section{INFINITE MEMORY}

In this section, we analyze the spectrum of system (1.4) with kernel (1.6). Special attention would be paid to the distribution of the spectrum on the complex plane and the asymptotic behavior of the eigenvalues.

\subsection{System operator setup}

The following general formulation comes from [17] for general kernel satisfying

(g1) $g \in C^{2}(0, \infty) \cap C[0, \infty)$, and $g_{s} \in L^{1}(0, \infty)$;

(g2) $g>0, g_{s}<0, g_{s s}>0$ on $(0, \infty)$;

(g3) $-k g_{s} \leq g_{s s} \leq-K g_{s}$ on $(0, \infty)$ for some $k, K>0$;

(g4) $g(\infty)=0$.

It is easily seen that the special kernel (1.6) satisfies the above four conditions. Let

$$
y(x, t, s)=u(x, t)-u(x, t-s), v=u_{t} .
$$

Then

$$
y_{t}=u_{t}-y_{s}
$$

and

$$
y(\cdot, \cdot, 0)=0 .
$$

The energy function of the system (1.4) is given by

$$
E(t)=\frac{1}{2} \int_{0}^{1}\left(a\left|u_{x}(x, t)\right|^{2}+\left|u_{t}(x, t)\right|^{2}\right) \mathrm{d} x+\frac{1}{2} \int_{0}^{\infty}\left|g_{s}(s)\right| \int_{0}^{1} b\left|y_{x}(x, t, s)\right|^{2} \mathrm{~d} x \mathrm{~d} s .
$$


Let $W=H_{0}^{1}(0,1)$ with the inner product:

$$
\left\langle w_{1}, w_{2}\right\rangle=b \int_{0}^{1} w_{1}^{\prime}(x) \overline{w_{2}^{\prime}(x)} \mathrm{d} x, \quad \forall w_{1}, w_{2} \in W .
$$

Define the energy state Hilbert space

$$
\mathcal{H}=V \times H \times Y,
$$

where

$$
\begin{gathered}
V=H_{0}^{1}(0,1), \quad\|u\|_{V}^{2}=a \int_{0}^{1}\left|u^{\prime}(x)\right|^{2} \mathrm{~d} x, \\
H=L^{2}(0,1), \quad\|v\|_{H}^{2}=\int_{0}^{1}|v(x)|^{2} \mathrm{~d} x, \\
Y=L^{2}((0, \infty) ; W), \quad\|y\|_{Y}^{2}=\int_{0}^{\infty} \mid g_{s}(s)\|y\|_{W}^{2} \mathrm{~d} s .
\end{gathered}
$$

Define the system operator $\mathcal{A}: D(\mathcal{A})(\subset \mathcal{H}) \rightarrow \mathcal{H}$ as

$$
\left\{\begin{array}{l}
\mathcal{A} z=\left(v,\left(a u^{\prime}-b \int_{0}^{\infty} g_{s}(s) y^{\prime}(\cdot, s) \mathrm{d} s\right)^{\prime}, v-y_{s}\right), \forall z=(u, v, y) \in D(\mathcal{A}) \\
D(\mathcal{A})=\left\{z \in \mathcal{H} \mid v \in V, y_{s} \in Y, y(\cdot, 0)=0, a u^{\prime}-b \int_{0}^{\infty} g_{s}(s) y^{\prime}(\cdot, s) \mathrm{d} s \in H^{1}(0,1)\right\} .
\end{array}\right.
$$

Then system (1.4) can be formulated as an abstract evolution equation in $\mathcal{H}$ ([17]):

$$
\frac{\mathrm{d}}{\mathrm{d} t} z(t)=\mathcal{A} z(t), \quad z(0)=z_{0},
$$

where $z(t)=\left(u(\cdot, t), u_{t}(\cdot, t), y(\cdot, t, \cdot)\right)$ is the state variable and $z_{0}(x)=\left(u_{0}(x, 0), u_{1}(x, 0), u_{0}(x, 0)-u_{0}(x,-s)\right)$ is the initial value.

Proposition 2.1 below justifies $\mathcal{A}^{*}$, the adjoint operator of $\mathcal{A}$. The proof is straightforward and we omit it in detail.

Proposition 2.1. Let $\mathcal{A}$ be defined by (2.6). Then its adjoint $\mathcal{A}^{*}$ has the following form:

$$
\left\{\begin{array}{l}
\mathcal{A}^{*} z=\left(-v,-\left(a u^{\prime}-b \int_{0}^{\infty} g_{s}(s) y^{\prime}(\cdot, s) \mathrm{d} s\right)^{\prime},-\left(v-y_{s}-\frac{g_{s s}(s)}{g_{s}(s)} y\right)\right), \quad z=(u, v, y), \\
D\left(\mathcal{A}^{*}\right)=\left\{z \in \mathcal{H} \mid u, v \in V, y, y_{s} \in Y, y(\cdot, 0)=0, a u^{\prime}-b \int_{0}^{\infty} g_{s}(s) y^{\prime}(\cdot, s) \mathrm{d} s \in H^{1}(0,1)\right\} .
\end{array}\right.
$$

The next Lemma 2.2 comes from Lemma 2.1 in [15].

Lemma 2.2. Suppose that $y \in Y, \operatorname{Re} \lambda>-\frac{k}{2}, g$ satisfies conditions (g1)-g(4),

$$
h(s)=\int_{0}^{s} \mathrm{e}^{-\lambda(s-\tau)} y(\tau) \mathrm{d} \tau .
$$

Then

(i) $h \in Y \cap C([0, \infty), W), h_{s} \in Y$, and

$$
\|h\|_{Y}^{2} \leq \frac{1}{\delta}(2 \operatorname{Re} \lambda+k-\delta)^{-1}\|y\|_{Y}^{2} \text { for } \delta \in(0,2 \operatorname{Re} \lambda+k) ;
$$


(ii)

$$
\operatorname{Re} \int_{0}^{\infty} g_{s}(s)\left\langle h_{s}(s), h(s)\right\rangle_{W} \mathrm{~d} s \leq-\frac{k}{2}\|h\|_{Y}^{2} .
$$

It was explained shortly in [17] that $\mathcal{A}$ is invertible and generates a $C_{0}$-semigroup. Here, we give a simple proof.

Proposition 2.3. Let $\mathcal{A}$ be defined by (2.6). Then $\mathcal{A}^{-1}$ is given by

$$
\mathcal{A}^{-1}\left(\begin{array}{l}
u \\
v \\
y
\end{array}\right)(x, s)=\left(\begin{array}{c}
h_{1}(x)+h_{2}(x)-\left[h_{1}(1)+h_{2}(1)\right] x \\
u(x) \\
u(x) s-\int_{0}^{s} y(x, \zeta) \mathrm{d} \zeta
\end{array}\right)
$$

where

$$
\left\{\begin{array}{l}
h_{1}(x)=\frac{b}{a} u(x) \int_{0}^{\infty} s g_{s}(s) \mathrm{d} s-\frac{b}{a} \int_{0}^{x}\left[\int_{0}^{\infty} g_{s}(s) \int_{0}^{s} y_{x}(\tau, \zeta) \mathrm{d} \zeta \mathrm{d} s\right] \mathrm{d} \tau \\
h_{2}(x)=\frac{1}{a} \int_{0}^{x}\left[\int_{0}^{\tau} v(\zeta) \mathrm{d} \zeta\right] \mathrm{d} \tau .
\end{array}\right.
$$

And hence $0 \in \rho(\mathcal{A})$, the resolvent set of $\mathcal{A}$. Moreover, $\mathcal{A}$ is dissipative, and thus $\mathcal{A}$ generates a $C_{0}$-semigroup of constructions $\mathrm{e}^{\mathcal{A} t}$ on $\mathcal{H}$.

Proof. Let $(u, v, y) \in \mathcal{H}$. By $\mathcal{A}(\widetilde{u}, \widetilde{v}, \widetilde{y})=(u, v, y)$, it has

$$
\left\{\begin{array}{l}
\widetilde{v}(x)=u(x) \\
\left(a \widetilde{u}_{x}(x)-b \int_{0}^{\infty} g_{s}(s) \widetilde{y}_{x}(x, s) \mathrm{d} s\right)_{x}=v(x) \\
\widetilde{v}(x)-\widetilde{y}_{s}(x, s)=y(x, s)
\end{array}\right.
$$

This together with the boundary conditions shows that $\widetilde{v}=u, \widetilde{y}=u s-\int_{0}^{s} y(\cdot, \zeta) \mathrm{d} \zeta$, and

$$
\left\{\begin{array}{l}
\left(a \widetilde{u}^{\prime}(x)-b \int_{0}^{\infty} g_{s}(s)\left[u^{\prime}(x) s-\int_{0}^{s} y^{\prime}(x, \zeta) \mathrm{d} \zeta\right] \mathrm{d} s\right)^{\prime}=v(x), \\
\widetilde{u}(0)=\widetilde{u}(1)=0 .
\end{array}\right.
$$

A direct computation gives

$$
\widetilde{u}(x)=h_{1}(x)+h_{2}(x)+\frac{C}{a} x,
$$

where $h_{1}(x), h_{2}(x)$ are given by $(2.11)$, and $C$ is a constant to be determined. Using the boundary condition $\widetilde{u}(1)=0$ gives

$$
C=-a\left[h_{1}(1)+h_{2}(1)\right]
$$

Therefore

$$
\widetilde{u}(x)=h_{1}(x)+h_{2}(x)-\left[h_{1}(1)+h_{2}(1)\right] x .
$$

By Lemma 2.2 , it has $\widetilde{y} \in Y$. And hence, $(\widetilde{u}, \widetilde{v}, \widetilde{y}) \in D(\mathcal{A}),(2.10)$ holds. 
By Lemma 2.2, for each $z=(u, v, y) \in D(\mathcal{A})$, it has

$$
\begin{aligned}
\operatorname{Re}\langle\mathcal{A} z, z\rangle= & \left.\operatorname{Re}\left\langle\left(v,\left(a u^{\prime}-b \int_{0}^{\infty} g_{s}(s) y^{\prime}(\cdot, s) \mathrm{d} s\right)^{\prime}, v-y_{s}\right)\right),(u, v, y)\right\rangle \\
= & \operatorname{Re}\left\{\int_{0}^{1} a v^{\prime}(x) \overline{u^{\prime}(x)} \mathrm{d} x+\int_{0}^{1}\left(a u^{\prime}(x)-b \int_{0}^{\infty} g_{s}(s) y^{\prime}(x, s) \mathrm{d} s\right)^{\prime} \overline{v(x)} \mathrm{d} x\right. \\
& \left.+\int_{0}^{\infty}\left|g_{s}(s)\right| \int_{0}^{1} b\left(v(x)-y_{s}(x, s)\right)^{\prime} \overline{y^{\prime}(x, s)} \mathrm{d} x \mathrm{~d} s\right\} \\
= & b \operatorname{Re} \int_{0}^{1} \int_{0}^{\infty} g_{s}(s) y_{s}^{\prime}(x, s) \overline{y^{\prime}(x, s)} \mathrm{d} s \mathrm{~d} x \\
\leq & -\frac{k}{2}\|y\|_{Y}^{2} .
\end{aligned}
$$

Therefore, $\mathcal{A}$ is dissipative. By the Lumer-Phillips theorem, $\mathcal{A}$ generates a $C_{0}$-semigroup of contractions on $\mathcal{H}$. The proof is complete.

\subsection{Spectral analysis for system operator}

In this subsection, we analyze the spectrum of $\mathcal{A}$ with the kernel (1.6). Firstly, consider the eigenvalue problem. Suppose $\mathcal{A} z=\lambda z$ for $0 \neq \lambda \in \mathbb{C}$ and $0 \neq z=(u, v, y) \in D(\mathcal{A})$. Then

$$
\left\{\begin{array}{l}
v(x)=\lambda u(x) \\
\left(a u^{\prime}(x)-b \int_{0}^{\infty} g_{s}(s) y^{\prime}(x, s) \mathrm{d} s\right)^{\prime}=\lambda v(x), \\
v(x)-y_{s}(x, s)=\lambda y(x, s), \\
u(0)=u(1)=0
\end{array}\right.
$$

From the third equation of $(2.12)$ and $y(\cdot, 0)=0$, we have

$$
y(x, s)=\frac{1}{\lambda}\left(1-\mathrm{e}^{-\lambda s}\right) v(x) .
$$

We claim that $v$ can not be identical to a constant. Actually, if this is the case, it follows from (2.12) that $(u, v, y)=0$. Hence $y \notin Y$ for any $\operatorname{Re} \lambda \leq-\frac{b_{1}}{2}$. Therefore,

$$
\sigma_{p}(\mathcal{A}) \subset D_{1}=\left\{\lambda \in \mathbb{C} \mid-\frac{b_{1}}{2}<\operatorname{Re} \lambda<0\right\},
$$

where $\sigma_{p}(\mathcal{A})$ denotes, as usual, the set of point spectrum of $\mathcal{A}$. By this fact, we always assume that $\lambda \in D_{1}$ when we mention the eigenvalues of $\mathcal{A}$ in what follows. Collecting these facts just mentioned, we find, from (2.12) and (2.13), that $\lambda \in \sigma_{p}(\mathcal{A})$ if and only if $(\lambda, u), u \neq 0$, satisfies

$$
\left\{\begin{array}{l}
\left(a+b \sum_{j=1}^{N} a_{j}-b \sum_{j=1}^{N} \frac{a_{j} b_{j}}{\lambda+b_{j}}\right) u^{\prime \prime}(x)-\lambda^{2} u(x)=0, \\
u(0)=u(1)=0 .
\end{array}\right.
$$


Lemma 2.4. Let $\mathcal{A}$ be defined by (2.6) and

$$
p(\lambda)=a+b \sum_{j=1}^{N} a_{j}-b \sum_{j=1}^{N} \frac{a_{j} b_{j}}{\lambda+b_{j}} .
$$

Then there exists a unique solution $\lambda_{c} \in\left\{\lambda \mid-b_{1}<\operatorname{Re} \lambda<0\right\}$ to $p(\lambda)=0$. Moreover, $\lambda_{c}$ is real, and

$$
\lambda_{c} \notin \sigma_{p}(\mathcal{A}) .
$$

Proof. Obviously, for any $j=1,2, \ldots, N, \lambda=-b_{j}$ is not the zero point of $p(\lambda)$. Thus, $p(\lambda)=0$ is equivalent to $\widetilde{p}(\lambda)=0$, where

$$
\widetilde{p}(\lambda)=p(\lambda) \prod_{j=1}^{N}\left(\lambda+b_{j}\right)=\left(a+b \sum_{j=1}^{N} a_{j}\right) \prod_{j=1}^{N}\left(\lambda+b_{j}\right)-b \sum_{j=1}^{N} a_{j} b_{j} \prod_{k=1, k \neq j}^{N}\left(\lambda+b_{k}\right) .
$$

However, $\widetilde{p}(\lambda)$ is an $N$-th order polynomial, and hence there are at most $N$ number of zeros for $p(\lambda)$. Now we find these zeros. Notice that $p(\lambda)$ is continues in $\left(\cup_{j=1}^{N-1}\left(-b_{j+1},-b_{j}\right)\right) \cup\left(-b_{1}, \infty\right)$, and

$$
\lim _{\lambda \rightarrow-b_{j}^{-}} p(\lambda)=+\infty, \quad \lim _{\lambda \rightarrow-b_{j}^{+}} p(\lambda)=-\infty, \quad p(0)>0, \quad j=1,2, \ldots, N .
$$

It follows that there exists a solution to $p(\lambda)=0$ in $\left(-b_{j+1},-b_{j}\right), j=0,1,2, \ldots, N-1$, here we set $b_{0}=0$. Moreover, when $\lambda_{c}>-\frac{b_{1}}{2}$ and $p(\lambda)=0$, it follows from (2.15) that $u \equiv 0$. This together with (2.12) gives $(u, v, y)=0$. Hence $(2.17)$ is valid. The proof is complete.

By Lemma 2.4, the eigenvalue problem (2.15) can be written as

$$
\left\{\begin{array}{l}
u^{\prime \prime}(x)=\frac{\lambda^{2}}{p(\lambda)} u(x), \\
u(0)=u(1)=0 .
\end{array}\right.
$$

The nonzero solution of $(2.18)$ is found to be

$$
u(x)=\mathrm{e}^{\sqrt{\frac{\lambda^{2}}{p(\lambda)}} x}-\mathrm{e}^{-\sqrt{\frac{\lambda^{2}}{p(\lambda)}} x},
$$

where $\lambda$ satisfies

$$
\mathrm{e}^{\sqrt{\frac{\lambda^{2}}{p(\lambda)}}}-\mathrm{e}^{-\sqrt{\frac{\lambda^{2}}{p(\lambda)}}}=0
$$

That is

$$
\mathrm{e}^{2 \sqrt{\frac{\lambda^{2}}{p(\lambda)}}}=1
$$

or

$$
\frac{\lambda^{2}}{p(\lambda)}=-n^{2} \pi^{2}, n=1,2, \ldots
$$

Substituting (2.21) into (2.19) gives the corresponding eigenfunction $\left(u(x), \lambda u(x),\left(1-\mathrm{e}^{-\lambda s}\right) u(x)\right)$, where

$$
u(x)=\sin n \pi x,
$$

for some $n \in \mathbb{N}^{+}$. 
Set

$$
\widetilde{a}=a+b \sum_{j=1}^{N} a_{j}
$$

When $|\lambda|$ is large enough, since

$$
\frac{\lambda^{2}}{p(\lambda)}=\frac{1}{\widetilde{a}}\left(\lambda^{2}+\frac{b}{\widetilde{a}} \sum_{j=1}^{N} a_{j} b_{j} \lambda-\frac{b}{\widetilde{a}} \sum_{j=1}^{N} a_{j} b_{j}^{2}+\frac{b^{2}}{\widetilde{a}^{2}}\left(\sum_{j=1}^{N} a_{j} b_{j}\right)^{2}\right)+\mathcal{O}\left(|\lambda|^{-1}\right),
$$

we obtain that

$$
\lambda^{2}+\frac{b}{\widetilde{a}} \sum_{j=1}^{N} a_{j} b_{j} \lambda-\frac{b}{\widetilde{a}} \sum_{j=1}^{N} a_{j} b_{j}^{2}+\frac{b^{2}}{\widetilde{a}^{2}}\left(\sum_{j=1}^{N} a_{j} b_{j}\right)^{2}+\widetilde{a} n^{2} \pi^{2}+\mathcal{O}\left(|\lambda|^{-1}\right)=0 .
$$

Thus, the eigenvalues of $\mathcal{A}$ are found to be

$$
\lambda_{n}=-\frac{b}{2 \widetilde{a}} \sum_{j=1}^{N} a_{j} b_{j} \pm i \sqrt{\widetilde{a}} n \pi+\mathcal{O}\left(n^{-1}\right), \quad n \rightarrow \infty .
$$

When $\lambda \rightarrow \lambda_{c}, \mu=\lambda-\lambda_{c} \rightarrow 0$. Since

$$
\begin{aligned}
p(\lambda) & =a+b \sum_{j=1}^{N} a_{j}-b \sum_{j=1}^{N} \frac{a_{j} b_{j}}{\lambda+b_{j}} \\
& =a+b \sum_{j=1}^{N} a_{j}-b \sum_{j=1}^{N} \frac{a_{j} b_{j}}{\lambda_{c}+b_{j}} \frac{1}{1+\frac{\left(\lambda-\lambda_{c}\right)}{\lambda_{c}+b_{j}}} \\
& =\mu b \sum_{j=1}^{N} a_{j} b_{j}\left[\frac{1}{\left(\lambda_{c}+b_{j}\right)^{2}}-\frac{\mu}{\left(\lambda_{c}+b_{j}\right)^{3}}+\mathcal{O}\left(\mu^{2}\right)\right],
\end{aligned}
$$

it has

$$
\begin{aligned}
\frac{\lambda^{2}}{p(\lambda)} & =\frac{\lambda_{c}^{2}+2 \lambda_{c} \mu+\mu^{2}}{p(\lambda)} \\
& =\frac{1}{\mu} \frac{\lambda_{c}^{2}}{\sum_{j=1}^{N} \frac{b a_{j} b_{j}}{\left(\lambda_{c}+b_{j}\right)^{2}}} \cdot\left(1+\frac{2}{\lambda_{c}} \mu+\frac{1}{\lambda_{c}^{2}} \mu^{2}\right) \cdot\left(1-\frac{\sum_{j=1}^{N} \frac{a_{j} b_{j}}{\left(\lambda_{c}+b_{j}\right)^{3}}}{\sum_{j=1}^{N} \frac{a_{j} b_{j}}{\left(\lambda_{c}+b_{j}\right)^{2}}} \mu+\mathcal{O}\left(\mu^{2}\right)\right)^{-1} \\
& =\frac{1}{\mu} \frac{\lambda_{c}^{2}}{\sum_{j=1}^{N} \frac{b a_{j} b_{j}}{\left(\lambda_{c}+b_{j}\right)^{2}}} \cdot\left(1+\frac{2}{\lambda_{c}} \mu+\frac{1}{\lambda_{c}^{2}} \mu^{2}\right) \cdot\left(1+\frac{\sum_{j=1}^{N} \frac{a_{j} b_{j}}{\left(\lambda_{c}+b_{j}\right)^{3}}}{\sum_{j=1}^{N} \frac{a_{j} b_{j}}{\left(\lambda_{c}+b_{j}\right)^{2}}} \mu\right)+\mathcal{O}(\mu) \\
& =\frac{1}{\mu} \frac{\lambda_{c}^{2}}{\Delta}\left[1+\left(\frac{2}{\lambda_{c}}+\frac{\Delta_{1}}{\Delta}\right) \mu\right]+\mathcal{O}(\mu),
\end{aligned}
$$

where

$$
\Delta=\sum_{j=1}^{N} \frac{b a_{j} b_{j}}{\left(\lambda_{c}+b_{j}\right)^{2}}, \quad \Delta_{1}=\sum_{j=1}^{N} \frac{a_{j} b_{j}}{\left(\lambda_{c}+b_{j}\right)^{3}} .
$$


This together with (2.21) yields

$$
\frac{1}{\mu} \frac{\lambda_{c}^{2}}{\Delta}\left[1+\left(\frac{2}{\lambda_{c}}+\frac{\Delta_{1}}{\Delta}\right) \mu\right]+\mathcal{O}(\mu)=-n^{2} \pi^{2}, n \rightarrow \infty .
$$

Thus

or

$$
\mu_{n}=-\frac{1}{n^{2} \pi^{2}} \frac{\lambda_{c}^{2}}{\Delta}+\mathcal{O}\left(n^{-3}\right), \quad n \rightarrow \infty
$$

$$
\lambda_{n}=\lambda_{c}-\frac{1}{n^{2} \pi^{2}} \frac{\lambda_{c}^{2}}{\Delta}+\mathcal{O}\left(n^{-3}\right), \quad n \rightarrow \infty .
$$

We summarize these results as Theorem 2.5 following.

Theorem 2.5. Let $\mathcal{A}$ be defined by (2.6). Then the eigenvalues of $\mathcal{A}$ must be located inside of $D_{1}$ that is given by (2.14). The eigenfunction corresponding to $\lambda$ is $\left(u(x), \lambda u(x),\left(1-\mathrm{e}^{-\lambda s}\right) u(x)\right)$ with

$$
u(x)=\sin n \pi x,
$$

for some $n \in \mathbb{N}^{+}$. More precisely,

(i) When $\lambda_{c}>-\frac{b_{1}}{2}$, where $\lambda_{c}$ is given in Lemma 2.4, there is a sequence of eigenvalues $\left\{\lambda_{n}\right\}$ of $\mathcal{A}$, which have the following asymptotic expression:

$$
\lambda_{n}=\lambda_{c}-\frac{1}{n^{2} \pi^{2}} \frac{\lambda_{c}^{2}}{\Delta}+\mathcal{O}\left(n^{-3}\right), \quad n \rightarrow \infty,
$$

where $\Delta$ is given by (2.24). Furthermore, the corresponding eigenfunctions $\left(u_{n}(x), \lambda_{n} u_{n}(x),\left(1-\mathrm{e}^{-\lambda_{n} s}\right) u_{n}(x)\right)$ are of the form:

$$
u_{n}(x)=\sin n \pi x, \quad n \rightarrow \infty .
$$

(ii) When $|\lambda| \rightarrow \infty$ and

$$
-\frac{b}{2 \widetilde{a}} \sum_{j=1}^{N} a_{j} b_{j}>-\frac{b_{1}}{2}
$$

the eigenvalues of $\mathcal{A}$ have the following asymptotic expressions:

$$
\lambda_{n}=-\frac{b}{2 \widetilde{a}} \sum_{j=1}^{N} a_{j} b_{j} \pm i \sqrt{\widetilde{a}} n \pi+\mathcal{O}\left(n^{-1}\right), \quad n \rightarrow \infty,
$$

where $\widetilde{a}$ is given by (2.23). In particular,

$$
\operatorname{Re} \lambda_{n} \rightarrow-\frac{b}{2 \widetilde{a}} \sum_{j=1}^{N} a_{j} b_{j}<0, \quad n \rightarrow \infty,
$$

that is, $\operatorname{Re} \lambda=-\frac{b}{2 a} \sum_{j=1}^{N} a_{j} b_{j}$ is the asymptote of the eigenvalues specified by (2.28). Furthermore, the corresponding eigenfunctions $\left(u_{n}(x), \lambda_{n} u_{n}(x),\left(1-\mathrm{e}^{-\lambda_{n} s}\right) u_{n}(x)\right)$ satisfy $(2.27)$.

Theorem 2.6. Let $\mathcal{A}$ be defined by (2.6), and $\lambda_{c}$ be given in Lemma 2.4. Then

$$
\sigma(\mathcal{A})=\sigma_{p}(\mathcal{A}) \cup\left\{\lambda_{c}\right\} \cup\left\{\lambda \mid \operatorname{Re} \lambda \leq-\frac{b_{1}}{2}\right\} .
$$


Proof. Let $\lambda \notin \sigma_{p}(\mathcal{A})$. If $\lambda=0$, by Proposition 2.3, $\lambda \in \rho(\mathcal{A})$. So we need only consider the case of $\lambda \neq 0$. For any $\widetilde{z}=(\widetilde{u}, \widetilde{v}, \widetilde{y}) \in \mathcal{H}$. Solve $(\lambda I-\mathcal{A}) z=\widetilde{z}$ for $z=(u, v, y)$, that is,

$$
\left\{\begin{array}{l}
\lambda u(x)-v(x)=\widetilde{u}(x) \\
\lambda v(x)-\left(a u^{\prime}(x)-b \int_{0}^{\infty} g_{s}(s) y^{\prime}(x, s) \mathrm{d} s\right)^{\prime}=\widetilde{v}(x) \\
\lambda y(x, s)-\left(v(x)-y_{s}(x, s)\right)=\widetilde{y}(x, s) \\
u(0)=u(1)=0
\end{array}\right.
$$

to get

$$
\left\{\begin{array}{l}
v(x)=\lambda u(x)-\widetilde{u}(x), \\
y(x, s)=\frac{1}{\lambda}\left(1-\mathrm{e}^{-\lambda s}\right) v(x)+\mathrm{e}^{-\lambda s} \int_{0}^{s} \mathrm{e}^{\lambda \tau} \widetilde{y}(x, \tau) \mathrm{d} \tau
\end{array}\right.
$$

and

$$
\left\{\begin{array}{l}
\left(a u^{\prime}(x)-b \int_{0}^{\infty} g_{s}(s) y^{\prime}(x, s) \mathrm{d} s\right)^{\prime}-\lambda^{2} u(x)+\lambda \widetilde{u}(x)+\widetilde{v}(x)=0, \\
u(0)=u(1)=0
\end{array}\right.
$$

There are three cases:

Case I: $\operatorname{Re} \lambda \leq-\frac{b_{1}}{2}$. We claim that $\lambda \in \sigma(\mathcal{A})$. In fact, take

$$
\widetilde{z}=(\widetilde{u}, \widetilde{v}, \widetilde{y})=(0, \widetilde{v}, 0), \quad \forall \widetilde{v} \in H, \widetilde{v} \neq 0 .
$$

It follows from (2.32) and (2.33) that

$$
\left\{\begin{array}{l}
v(x)=\lambda u(x), \\
y(x, s)=\left(1-\mathrm{e}^{-\lambda s}\right) u(x), \\
\left(a u^{\prime}(x)-b \int_{0}^{\infty} g_{s}(s)\left(1-\mathrm{e}^{-\lambda s}\right) u^{\prime}(x) \mathrm{d} s\right)^{\prime}-\lambda^{2} u(x)+\widetilde{v}(x)=0, \\
u(0)=u(1)=0 .
\end{array}\right.
$$

If (2.34) admits a solution, it must have $y \in Y$. This together with $\operatorname{Re} \lambda \leq-\frac{b_{1}}{2}$ shows that $u^{\prime} \equiv 0$. Thus, $u \equiv 0$, and so $\widetilde{v} \equiv 0$. This is a contradiction. Therefore, there is no solution to equation (2.34), which means that $\lambda \in \sigma(\mathcal{A})$.

Case II: $\operatorname{Re} \lambda>-\frac{b_{1}}{2}$ and $\lambda \neq \lambda_{c}$. We show that $\lambda \in \rho(\mathcal{A})$. By Lemma 2.2, it has $y \in Y$. (2.33) is equivalent to

$$
\left\{\begin{array}{l}
\eta^{\prime}(x)-\lambda^{2} u(x)+\lambda \widetilde{u}(x)+\widetilde{v}(x)=0, \\
\eta(x)=p(\lambda) u^{\prime}(x)+\frac{1}{\lambda}(a-p(\lambda)) \widetilde{u}^{\prime}(x)-b \int_{0}^{\infty} g_{s}(s)\left[\int_{0}^{s} \mathrm{e}^{-\lambda(s-\tau)} \widetilde{y}^{\prime}(x, \tau) \mathrm{d} \tau\right] \mathrm{d} s, \\
u(0)=u(1)=0 .
\end{array}\right.
$$

We write above equation as the following first order system of differential equations

$$
\left\{\begin{array}{l}
\frac{\mathrm{d}}{\mathrm{d} x}\left(\begin{array}{c}
u(x) \\
\eta(x)
\end{array}\right)=\left(\begin{array}{cc}
0 & \frac{1}{p(\lambda)} \\
\lambda^{2} & 0
\end{array}\right)\left(\begin{array}{l}
u(x) \\
\eta(x)
\end{array}\right)+\left(\begin{array}{c}
\frac{1}{p(\lambda)} U(x) \\
-\lambda \widetilde{u}(x)-\widetilde{v}(x)
\end{array}\right), \\
u(0)=u(1)=0
\end{array}\right.
$$


where

$$
U(x)=-\frac{1}{\lambda}(a-p(\lambda)) \widetilde{u}^{\prime}(x)+b \int_{0}^{\infty} g_{s}(s)\left[\int_{0}^{s} \mathrm{e}^{-\lambda(s-\tau)} \widetilde{y}^{\prime}(x, \tau) \mathrm{d} \tau\right] \mathrm{d} s .
$$

Let

$$
A(\lambda)=\left(\begin{array}{cc}
0 & \frac{1}{p(\lambda)} \\
\lambda^{2} & 0
\end{array}\right)
$$

Then

$$
\mathrm{e}^{A(\lambda) x}=\left(\begin{array}{ll}
a_{11}(\lambda, x) & a_{12}(\lambda, x) \\
a_{21}(\lambda, x) & a_{22}(\lambda, x)
\end{array}\right)
$$

where

$$
\left\{\begin{array} { l } 
{ a _ { 1 1 } ( \lambda , x ) = \operatorname { c o s h } ( \frac { \lambda } { \sqrt { p ( \lambda ) } } x ) , } \\
{ a _ { 2 1 } ( \lambda , x ) = \lambda \sqrt { p ( \lambda ) } \operatorname { s i n h } ( \frac { \lambda } { \sqrt { p ( \lambda ) } } x ) , }
\end{array} \left\{\begin{array}{l}
a_{12}(\lambda, x)=\frac{1}{\lambda \sqrt{p(\lambda)}} \sinh \left(\frac{\lambda}{\sqrt{p(\lambda)}} x\right), \\
a_{22}(\lambda, x)=\cosh \left(\frac{\lambda}{\sqrt{p(\lambda)}} x\right) .
\end{array}\right.\right.
$$

The general solution of (2.36) is given by

$$
\left(\begin{array}{l}
u(x) \\
\eta(x)
\end{array}\right)=\mathrm{e}^{A(\lambda) x}\left(\begin{array}{l}
u(0) \\
\eta(0)
\end{array}\right)-\int_{0}^{x} \mathrm{e}^{A(\lambda)(x-\gamma)}\left(\begin{array}{c}
\frac{1}{p(\lambda)} U(\gamma) \\
-\lambda \widetilde{u}(\gamma)-\widetilde{v}(\gamma)
\end{array}\right) \mathrm{d} \gamma
$$

By $u(0)=0$, it has,

$$
u(x)=a_{12}(\lambda, x) \eta(0)-\int_{0}^{x}\left[\frac{1}{p(\lambda)} a_{11}(\lambda, x-\gamma) U(\gamma)+a_{12}(\lambda, x-\gamma)(-\lambda \widetilde{u}(\gamma)-\widetilde{v}(\gamma))\right] \mathrm{d} \gamma
$$

and

$$
\eta(x)=a_{22}(\lambda, x) \eta(0)-\int_{0}^{x}\left[\frac{1}{p(\lambda)} a_{21}(\lambda, x-\gamma) U(\gamma)+a_{22}(\lambda, x-\gamma)(-\lambda \widetilde{u}(\gamma)-\widetilde{v}(\gamma))\right] \mathrm{d} \gamma
$$

Since $\lambda \notin \sigma_{p}(\mathcal{A})$, it follows from (2.20) that

$$
a_{12}(\lambda, 1)=\frac{1}{\lambda \sqrt{p(\lambda)}} \sinh \left(\frac{\lambda}{\sqrt{p(\lambda)}}\right) \neq 0
$$

By the boundary condition $u(1)=0$, it has

$$
\eta(0)=\frac{1}{a_{12}(\lambda, 1)} \int_{0}^{1}\left[\frac{1}{p(\lambda)} a_{11}(\lambda, 1-\gamma) U(\gamma)+a_{12}(\lambda, 1-\gamma)(-\lambda \widetilde{u}(\gamma)-\widetilde{v}(\gamma))\right] \mathrm{d} \gamma
$$

Hence $u$ is uniquely determined by (2.38). By the second equation of $(2.35)$ and $(2.39)$, it has $u^{\prime} \in L^{2}(0,1)$. This together with $(2.32)$ shows that $(\lambda I-\mathcal{A})^{-1}$ exists and is bounded, or $\lambda \in \rho(\mathcal{A})$.

Case III: $\lambda=\lambda_{c}>-\frac{b_{1}}{2}$. In this case, it follows from (2.33) that

$$
\left\{\begin{array}{l}
u(x)=\frac{1}{\lambda^{2}}\left[\lambda \widetilde{u}(x)+\widetilde{v}(x)-U^{\prime}(x)\right] \\
u(0)=u(1)=0
\end{array}\right.
$$


where $U$ is given by (2.37). Since $\widetilde{u} \in H_{0}^{1}(0,1),(2.41)$ means that (2.31) admits a solution if and only if $U$ is differentiable and

$$
\widetilde{v}(0)-U^{\prime}(0)=\widetilde{v}(1)-U^{\prime}(1)=0 .
$$

Thus $\lambda \notin \rho(\mathcal{A})$.

Combing all these cases completes the proof.

\section{Finite MEMORY}

In this section, we turn to the system (1.5) with kernel (1.6). We analyze the spectrum of the system operator first, and then prove the Riesz basis property for the system. The idea comes from [23] but the result is different, particularly for the basis property.

\subsection{System operator setup}

In what follows, we always assume (1.8). Set

$$
h_{j}(x, t)=a_{j} b_{j} \int_{0}^{t} \mathrm{e}^{-b_{j}(t-s)} u_{x}(x, s) \mathrm{d} s, j=1,2, \ldots, N .
$$

Then it has

$$
\left\{\begin{array}{l}
\left(h_{j}\right)_{t}(x, t)=a_{j} b_{j} u_{x}(x, t)-b_{j} h_{j}(x, t), \\
\left(h_{j}\right)_{x}(x, t)=a_{j} b_{j} \int_{0}^{t} \mathrm{e}^{-b_{j}(t-s)} u_{x x}(x, s) \mathrm{d} s \\
h_{j}(x, 0)=0 .
\end{array}\right.
$$

Thus we can rewrite the system (1.5),(1.6) as

$$
\left\{\begin{array}{l}
u_{t t}(x, t)=\left(a u_{x}(x, t)-b \sum_{j=1}^{N} h_{j}(x, t)\right)_{x}, x \in(0,1), t>0 \\
\left(h_{j}\right)_{t}(x, t)=a_{j} b_{j} u_{x}(x, t)-b_{j} h_{j}(x, t), j=1,2, \ldots, N \\
u(0, t)=u(1, t)=0 \\
u(x, 0)=u_{0}(x), u_{t}(x, 0)=u_{1}(x), h_{j}(x, 0)=0, j=1,2, \ldots, N
\end{array}\right.
$$

The system energy is given by

$$
E(t)=\frac{1}{2} \int_{0}^{1}\left[a\left|u_{x}(x, t)\right|^{2}+\left|u_{t}(x, t)\right|^{2}+\sum_{j=1}^{N}\left|h_{j}(x, t)\right|^{2}\right] \mathrm{d} x .
$$

We consider the system (3.3) in the energy state Hilbert space $\mathcal{H}=H_{0}^{1}(0,1) \times\left(L^{2}(0,1)\right)^{N+1}$ with the inner product:

$$
\begin{aligned}
& \left\langle\left(u, v, h_{1}, \ldots, h_{N}\right),\left(\widetilde{u}, \widetilde{v}, \widetilde{h_{1}}, \ldots, \widetilde{h_{N}}\right)\right\rangle \\
& =\int_{0}^{1} a u^{\prime}(x) \overline{\widetilde{u}^{\prime}(x)} \mathrm{d} x+\int_{0}^{1} v(x) \overline{\widetilde{v}(x)} \mathrm{d} x+\sum_{j=1}^{N} \int_{0}^{1} h_{j}(x) \overline{\widetilde{h_{j}}(x)} \mathrm{d} x \\
& \quad \forall\left(u, v, h_{1}, \ldots, h_{N}\right),\left(\widetilde{u}, \widetilde{v}, \widetilde{h_{1}}, \ldots, \widetilde{h_{N}}\right) \in \mathcal{H} .
\end{aligned}
$$


Define the system operator $\mathcal{B}: D(\mathcal{B})(\subset \mathcal{H}) \rightarrow \mathcal{H}$ as

$$
\left\{\begin{array}{c}
\mathcal{B}\left(\begin{array}{c}
u \\
v \\
h_{1} \\
\vdots \\
h_{N}
\end{array}\right)^{\top}=\left(\begin{array}{c}
v \\
\left(a u^{\prime}-b \sum_{j=1}^{N} h_{j}\right)^{\prime} \\
a_{1} b_{1} u^{\prime}-b_{1} h_{1} \\
\vdots \\
a_{N} b_{N} u^{\prime}-b_{N} h_{N}
\end{array}\right)^{\top}, \\
\left.D(\mathcal{B})=\left\{\begin{array}{c}
u \\
v \\
h_{1} \\
\vdots \\
h_{N}
\end{array}\right) \mid \begin{array}{l}
u, v \in H_{0}^{1}(0,1), \\
h_{j} \in L^{2}(0,1), j=1, \ldots, N, \\
a u^{\prime}-b \sum_{j=1}^{N} h_{j} \in H^{1}(0,1)
\end{array}\right\} .
\end{array}\right.
$$

Then (3.3) can be formulated into an abstract evolution equation in $\mathcal{H}$ :

$$
\frac{\mathrm{d}}{\mathrm{d} t} U(t)=\mathcal{B} U(t), \quad U(0)=U_{0},
$$

where $U(t)=\left(u(\cdot, t), u_{t}(\cdot, t), h_{1}(\cdot, t), \ldots, h_{N}(\cdot, t)\right)$ is the state variable and $U_{0}=\left(u_{0}(\cdot), u_{1}(\cdot), 0, \ldots, 0\right)$ is the initial value.

Lemma 3.1. Let $\mathcal{B}$ be defined by (3.6). Then $0 \in \rho(\mathcal{B})$.

Proof. Let $\widetilde{U}=\left(\widetilde{u}, \widetilde{v}, \widetilde{h_{1}}, \ldots, \widetilde{h_{N}}\right) \in \mathcal{H}$. Solve $\mathcal{B} U=\widetilde{U}$ for $U=\left(u, v, h_{1}, \ldots, h_{N}\right)$, that is

$$
\left\{\begin{array}{l}
v(x)=\widetilde{u}(x) \\
\left(a u^{\prime}(x)-b \sum_{j=1}^{N} h_{j}(x)\right)^{\prime}=\widetilde{v}(x), \\
a_{j} b_{j} u^{\prime}(x)-b_{j} h_{j}(x)=\widetilde{h_{j}}(x), \quad j=1,2, \ldots, N \\
u(0)=u(1)=0
\end{array}\right.
$$

to give

$$
v(x)=\widetilde{u}(x), \quad h_{j}(x)=a_{j} u^{\prime}(x)-\frac{1}{b_{j}} \widetilde{h_{j}}(x), j=1,2, \ldots, N
$$

and

$$
\left(a-b \sum_{j=1}^{N} a_{j}\right) u^{\prime}(x)+b \sum_{j=1}^{N} \frac{1}{b_{j}} \widetilde{h_{j}}(x)=\int_{0}^{x} \widetilde{v}(\tau) \mathrm{d} \tau+C_{1},
$$

where $C_{1}$ is a constant to be determined. By the boundary condition $u(0)=0$, it follows from (3.10) that

$$
u(x)=-\frac{b}{A} \int_{0}^{x} \sum_{j=1}^{N} \frac{1}{b_{j}} \widetilde{h_{j}}(\tau) \mathrm{d} \tau+\frac{1}{A} \int_{0}^{x} \int_{0}^{s} \widetilde{v}(\tau) \mathrm{d} \tau \mathrm{d} s+\frac{C_{1}}{A} x,
$$


where

$$
A=a-b \sum_{j=1}^{N} a_{j} .
$$

Using the other boundary condition $u(1)=0$, it gives

$$
C_{1}=b \int_{0}^{1} \sum_{j=1}^{N} \frac{1}{b_{j}} \widetilde{h_{j}}(x) \mathrm{d} x-\int_{0}^{1} \int_{0}^{s} \widetilde{v}(\tau) \mathrm{d} \tau \mathrm{d} s .
$$

This together with (3.9) and (3.11) gives the unique solution $U \in D(\mathcal{B})$ to equation (3.8). Hence $\mathcal{B}^{-1}$ exists and is bounded, or $0 \in \rho(\mathcal{B})$.

\subsection{Spectrum of system operator}

In this subsection, we consider the spectrum of $\mathcal{B}$. As in previous section, we first consider the eigenvalue problem. Suppose $\mathcal{B} U=\lambda U$ for $\lambda \in \mathbb{C}$ and $0 \neq U=\left(u, v, h_{1}, \ldots, h_{N}\right) \in D(\mathcal{B})$, that is,

$$
\left\{\begin{array}{l}
v(x)=\lambda u(x), \\
\left(a u^{\prime}(x)-b \sum_{j=1}^{N} h_{j}(x)\right)^{\prime}=\lambda v(x), \\
a_{j} b_{j} u^{\prime}(x)-b_{j} h_{j}(x)=\lambda h_{j}(x), \quad j=1,2, \ldots, N \\
u(0)=u(1)=0 .
\end{array}\right.
$$

Proposition 3.2. Let $\mathcal{B}$ be defined by (3.6). Then $\lambda=-b_{j}, j=1,2, \ldots, N$ are eigenvalues of $\mathcal{B}$, which corresponding to eigenfunctions $e_{j+2}, j=1,2, \ldots, N$ respectively, where $e_{j}$ is a constant function whose element is the $j$ th element of the canonical basis of $\mathbb{R}^{N+2}$. Moreover, each of these eigenvalues is algebraically simple.

Proof. We only give the proof for $\lambda=-b_{1}$ because other cases can be treated similarly. Let $\lambda=-b_{1}$ and $U=\left(u, v, h_{1}, \ldots, h_{N}\right) \in D(\mathcal{B})$. Since $\lambda=-b_{1},(3.13)$ becomes

$$
\left\{\begin{array}{l}
v(x)=-b_{1} u(x) \\
\left(a u^{\prime}(x)-b \sum_{j=1}^{N} h_{j}(x)\right)^{\prime}=-b_{1} v(x), \\
a_{1} b_{1} u^{\prime}(x)=0, \\
\left(b_{j}-b_{1}\right) h_{j}(x)=a_{j} b_{j} u^{\prime}(x), \quad j=2, \ldots, N, \\
u(0)=u(1)=0 .
\end{array}\right.
$$

This together with (1.7) yields

$$
u(x)=v(x)=h_{j}(x)=0, j=2, \ldots, N .
$$

By the second equation of (3.14), it has

$$
h_{1}^{\prime}(x)=0 .
$$


Therefor, $e_{3}$ is an eigenfunction of $\mathcal{B}$ corresponding to $-b_{1}$. Further computation of $\left(b_{1} I+\mathcal{B}\right) U_{1}=-e_{3}$, where $U_{1}=\left(\widetilde{u}, \widetilde{v}, \widetilde{h_{1}}, \ldots, \widetilde{h_{N}}\right) \in D(\mathcal{B})$, gives

$$
\left\{\begin{array}{l}
\widetilde{v}(x)=-b_{1} \widetilde{u}(x) \\
\left(a \widetilde{u}^{\prime}(x)-b \sum_{j=1}^{N} \widetilde{h_{j}}(x)\right)^{\prime}=-b_{1} \widetilde{v}(x), \\
a_{1} b_{1} \widetilde{u}^{\prime}(x)=-1, \\
\left(b_{j}-b_{1}\right) \widetilde{h_{j}}(x)=a_{j} b_{j} \widetilde{u}^{\prime}(x), \quad j=2, \ldots, N \\
\widetilde{u}(0)=\widetilde{u}(1)=0 .
\end{array}\right.
$$

(3.16) has no solution since otherwise, $\widetilde{u}$ satisfies

$$
a_{1} b_{1} \widetilde{u}^{\prime}(x)=-1, \quad \widetilde{u}(0)=\widetilde{u}(1)=0,
$$

which is impossible. This shows that $-b_{1}$ is algebraically simple. The proof is complete.

When $\lambda \neq-b_{j}, j=1,2, \ldots, N$, it follows from (3.13) that

$$
\left\{\begin{array}{l}
v(x)=\lambda u(x), \\
h_{j}(x)=\frac{a_{j} b_{j}}{\lambda+b_{j}} u^{\prime}(x), \quad j=1,2, \ldots, N
\end{array}\right.
$$

and $u$ satisfies

$$
\left\{\begin{array}{l}
\left(a-b \sum_{j=1}^{N} \frac{a_{j} b_{j}}{\lambda+b_{j}}\right) u^{\prime \prime}(x)=\lambda^{2} u(x), \\
u(0)=u(1)=0 .
\end{array}\right.
$$

The following Lemma 3.3 is straightforward.

Lemma 3.3. Let $\mathcal{B}$ be defined by (3.6) and

$$
\Lambda=\left\{\begin{array}{l|l}
\lambda \in \mathbb{C} & a-b \sum_{j=1}^{N} \frac{a_{j} b_{j}}{\lambda+b_{j}}=0
\end{array}\right\} .
$$

Then

$$
\Lambda \cap \sigma_{p}(\mathcal{B})=\emptyset .
$$

Lemma 3.4. Let $\mathcal{B}$ be defined by (3.6). $\Lambda$ is given by (3.19). Then

$$
\Lambda=\left\{\lambda_{c 1}, \lambda_{c 2}, \ldots, \lambda_{c N}\right\}
$$

where $\lambda_{c 1} \in\left(-b_{1}, 0\right)$, and $\lambda_{c k} \in\left(-b_{k},-b_{k-1}\right), k=2, \ldots, N$.

Proof. Since $-b_{j} \notin \Lambda, j=1,2, \ldots, N, p(\lambda)=0$ is equivalent to $q(\lambda)=0$, where

$$
p(\lambda)=a-b \sum_{j=1}^{N} \frac{a_{j} b_{j}}{\lambda+b_{j}}, \quad q(\lambda)=p(\lambda) \prod_{j=1}^{N}\left(\lambda+b_{j}\right) .
$$


However, $q(\lambda)$ is an $N$ th order polynomial, and hence there are at most $N$ number of zeros for $p(\lambda)$. Now we find all these zeros.

Since $p(\lambda)$ is continues in $\left(-b_{1}, \infty\right) \cup\left(\cup_{j=1}^{N-1}\left(-b_{j+1},-b_{j}\right)\right)$, by the fact

$$
\lim _{\lambda \rightarrow-b_{1}^{+}} p(\lambda)=-\infty
$$

and (1.8), we see that there exists a solution to $p(\lambda)=0$ in $\left(-b_{1}, 0\right)$. For any $j=1,2, \ldots, N-1$, it has

$$
\lim _{\lambda \rightarrow-b_{j+1}^{+}} p(\lambda)=-\infty, \quad \lim _{\lambda \rightarrow-b_{j}^{-}} p(\lambda)=+\infty .
$$

Therefore, there exists a solution to $p(\lambda)=0$ in $\left(-b_{j+1},-b_{j}\right)$. The proof is complete.

By Lemma 3.3, the eigenvalue problem (3.18) is equivalent to the following problem:

$$
\left\{\begin{array}{l}
u^{\prime \prime}(x)=\frac{\lambda^{2}}{p(\lambda)} u(x), \\
u(0)=u(1)=0,
\end{array}\right.
$$

where $p(\lambda)$ is given by (3.22). Hence

$$
u(x)=\mathrm{e}^{\sqrt{\frac{\lambda^{2}}{p(\lambda)}} x}-\mathrm{e}^{-\sqrt{\frac{\lambda^{2}}{p(\lambda)}} x} .
$$

By the boundary condition $u(1)=0,(3.23)$ has non-trivial solution if and only if

$$
\mathrm{e}^{\sqrt{\frac{\lambda^{2}}{p(\lambda)}}}-\mathrm{e}^{-\sqrt{\frac{\lambda^{2}}{p(\lambda)}}}=0 .
$$

That is

$$
\mathrm{e}^{2 \sqrt{\frac{\lambda^{2}}{p(\lambda)}}}=1
$$

which is equivalent to

$$
\frac{\lambda^{2}}{p(\lambda)}=-n^{2} \pi^{2}, n=1,2, \ldots
$$

Substituting (3.26) into (3.24), we obtain the eigenfunction $\left(u(x), \lambda u(x), \frac{a_{1} b_{1}}{\lambda+b_{1}} u^{\prime}(x), \ldots, \frac{a_{N} b_{N}}{\lambda+b_{N}} u^{\prime}(x)\right)$ corresponding to $\lambda$, where

$$
u(x)=\sin n \pi x,
$$

for some $n \in \mathbb{N}^{+}$.

When $|\lambda|$ is large enough, since

$$
\frac{\lambda^{2}}{p(\lambda)}=\frac{1}{a}\left(\lambda^{2}+\frac{b}{a} \sum_{j=1}^{N} a_{j} b_{j} \lambda-\frac{b}{a} \sum_{j=1}^{N} a_{j} b_{j}^{2}+\frac{b^{2}}{a^{2}}\left(\sum_{j=1}^{N} a_{j} b_{j}\right)^{2}\right)+\mathcal{O}\left(|\lambda|^{-1}\right),
$$

it has

$$
\lambda^{2}+\frac{b}{a} \sum_{j=1}^{N} a_{j} b_{j} \lambda-\frac{b}{a} \sum_{j=1}^{N} a_{j} b_{j}^{2}+\frac{b^{2}}{a^{2}}\left(\sum_{j=1}^{N} a_{j} b_{j}\right)^{2}+a n^{2} \pi^{2}+\mathcal{O}\left(|\lambda|^{-1}\right)=0 .
$$


Thus, the eigenvalues of $\mathcal{B}$ is this case are found to be

$$
\lambda_{n}=-\frac{b}{2 a} \sum_{j=1}^{N} a_{j} b_{j} \pm i \sqrt{a} n \pi+\mathcal{O}\left(n^{-1}\right), \quad n \rightarrow \infty .
$$

For any $\lambda_{c} \in \Lambda$, when $\lambda \rightarrow \lambda_{c}, \mu=\lambda-\lambda_{c} \rightarrow 0$. Notice that

$$
p(\lambda)=\mu b \sum_{j=1}^{N} a_{j} b_{j}\left[\frac{1}{\left(\lambda_{c}+b_{j}\right)^{2}}-\frac{\mu}{\left(\lambda_{c}+b_{j}\right)^{3}}+\mathcal{O}\left(\mu^{2}\right)\right] .
$$

We have

$$
\frac{\lambda^{2}}{p(\lambda)}=\frac{1}{\mu} \frac{\lambda_{c}^{2}}{\Delta}\left[1+\left(\frac{2}{\lambda_{c}}+\frac{\widetilde{\Delta}}{\Delta}\right) \mu\right]+\mathcal{O}(\mu)
$$

where

$$
\Delta=\sum_{j=1}^{N} \frac{b a_{j} b_{j}}{\left(\lambda_{c}+b_{j}\right)^{2}}, \quad \widetilde{\Delta}=\sum_{j=1}^{N} \frac{a_{j} b_{j}}{\left(\lambda_{c}+b_{j}\right)^{3}} .
$$

This together with (3.26) yields

$$
\frac{1}{\mu} \frac{\lambda_{c}^{2}}{\Delta}\left[1+\left(\frac{2}{\lambda_{c}}+\frac{\widetilde{\Delta}}{\Delta}\right) \mu\right]+\mathcal{O}(\mu)=-n^{2} \pi^{2}, n \rightarrow \infty .
$$

Thus,

$$
\mu_{n}=-\frac{1}{n^{2} \pi^{2}} \frac{\lambda_{c}^{2}}{\Delta}+\mathcal{O}\left(n^{-3}\right), \quad n \rightarrow \infty .
$$

Hence, the eigenvalues of $\mathcal{B}$ in this case are given by

$$
\lambda_{n}=\lambda_{c}-\frac{1}{n^{2} \pi^{2}} \frac{\lambda_{c}^{2}}{\Delta}+\mathcal{O}\left(n^{-3}\right), \quad n \rightarrow \infty .
$$

We summarize these results as Proposition 3.5 following.

Proposition 3.5. Let $\mathcal{B}$ be defined by (3.6), $\lambda$ be an eigenvalue of $\mathcal{B}$, satisfying $\lambda \neq-b_{j}, j=1,2, \ldots, N$. Then the eigenfunction corresponding to $\lambda$ is of the form

$$
\left(u(x), \lambda u(x), \frac{a_{1} b_{1}}{\lambda+b_{1}} u^{\prime}(x), \ldots, \frac{a_{N} b_{N}}{\lambda+b_{N}} u^{\prime}(x)\right)
$$

where

$$
u(x)=\sin n \pi x,
$$

for some $n \in \mathbb{N}^{+}$. Furthermore,

(i) For any $1 \leq k \leq N$, there is a sequence of eigenvalues $\left\{\lambda_{n k}\right\}$ of $\mathcal{B}$, which have the following asymptotic expressions:

$$
\lambda_{n k}=\lambda_{c k}-\frac{1}{n^{2} \pi^{2}} \frac{\lambda_{c k}^{2}}{\Delta_{k}}+\mathcal{O}\left(n^{-3}\right), \quad n \rightarrow \infty
$$

where

$$
\Delta_{k}=\sum_{j=1}^{N} \frac{b a_{j} b_{j}}{\left(\lambda_{c k}+b_{j}\right)^{2}}
$$


The corresponding eigenfunctions $\left(u_{n}(x), \lambda u_{n}(x), \frac{a_{1} b_{1}}{\lambda+b_{1}} u_{n}^{\prime}(x), \ldots, \frac{a_{N} b_{N}}{\lambda+b_{N}} u_{n}^{\prime}(x)\right)$ satisfy

$$
u_{n}(x)=\frac{1}{n \pi} \sin n \pi x, \quad n \rightarrow \infty .
$$

(ii) When $|\lambda| \rightarrow \infty$, the eigenvalues $\left\{\lambda_{n 0}, \overline{\lambda_{n 0}}\right\}$ of $\mathcal{B}$ have the following asymptotic expressions:

$$
\lambda_{n 0}=-\frac{b}{2 a} \sum_{j=1}^{N} a_{j} b_{j}+i \sqrt{a} n \pi+\mathcal{O}\left(n^{-1}\right), \quad n \rightarrow \infty,
$$

where $\overline{\lambda_{n 0}}$ denotes the complex conjugate of $\lambda_{n 0}$. In particular,

$$
\operatorname{Re} \lambda_{n 0} \rightarrow-\frac{b}{2 a} \sum_{j=1}^{N} a_{j} b_{j}<0, \quad n \rightarrow \infty
$$

that is, $\operatorname{Re} \lambda=-\frac{b}{2 a} \sum_{j=1}^{N} a_{j} b_{j}$ is the asymptote of the eigenvalues $\lambda_{n 0}$ given by (3.32). Furthermore, the corresponding eigenfunctions $\left(u_{n}(x), \lambda u_{n}(x), \frac{a_{1} b_{1}}{\lambda+b_{1}} u_{n}^{\prime}(x), \ldots, \frac{a_{N} b_{N}}{\lambda+b_{N}} u_{n}^{\prime}(x)\right)$ satisfy (3.31).

Combing Propositions 3.2 and 3.5, we obtain the following Theorem 3.6.

Theorem 3.6. Let $\mathcal{B}$ be defined by (3.6). Then

(i) $\mathcal{B}$ has the eigenvalues

$$
\left\{-b_{j}, j=1,2, \ldots, N\right\} \cup\left\{\lambda_{n 1}, \lambda_{n 2}, \ldots, \lambda_{n N}, \lambda_{n 0}, \overline{\lambda_{n 0}}, n \in \mathbb{N}^{+}\right\},
$$

where $\lambda_{n k}, k=1,2, \ldots, N$ and $\lambda_{n 0}$ have the asymptotic expressions (3.29) and (3.32), respectively.

(ii) The eigenfunction corresponding to $-b_{j}$ is $e_{j+2}$ for any $j=1,2, \ldots, N$.

(iii) The eigenfunctions corresponding to $\lambda_{n k}, k=1,2, \ldots, N$, are given by

$$
\begin{aligned}
U_{n k}(x)= & \left(\frac{1}{n \pi} \sin n \pi x, 0, \frac{a_{1} b_{1}}{\lambda_{n k}+b_{1}} \cos n \pi x, \ldots, \frac{a_{N} b_{N}}{\lambda_{n k}+b_{N}} \cos n \pi x\right) \\
& +\left(0, \mathcal{O}\left(n^{-1}\right), \ldots, \mathcal{O}\left(n^{-1}\right)\right), \quad n \rightarrow \infty .
\end{aligned}
$$

(iv) The eigenfunctions corresponding to $\lambda_{n 0}$ and $\overline{\lambda_{n 0}}$, are given by

$$
U_{n 0}(x)=\left(\frac{1}{n \pi} \sin n \pi x, i \sqrt{a} \sin n \pi x, 0, \ldots, 0\right)+\left(0, \mathcal{O}\left(n^{-1}\right), \ldots, \mathcal{O}\left(n^{-1}\right)\right), n \rightarrow \infty
$$

and

$$
\overline{U_{n 0}}(x)=\left(\frac{1}{n \pi} \sin n \pi x,-i \sqrt{a} \sin n \pi x, 0, \ldots, 0\right)+\left(0, \mathcal{O}\left(n^{-1}\right), \ldots, \mathcal{O}\left(n^{-1}\right)\right), n \rightarrow \infty
$$

respectively.

Concerning about $\sigma(\mathcal{B})$, we have the following Theorem 3.7.

Theorem 3.7. Let $\mathcal{B}$ be defined by (3.6). $\Lambda$ is given by (3.19). Then

$$
\sigma(\mathcal{B})=\Lambda \cup \sigma_{p}(\mathcal{B})
$$


Proof. Let $\lambda \notin \sigma_{p}(\mathcal{B})$. For any $\widetilde{U}=\left(\widetilde{u}, \widetilde{v}, \widetilde{h_{1}}, \ldots, \widetilde{h_{N}}\right) \in \mathcal{H}$. Solve $(\lambda I-\mathcal{B}) U=\widetilde{U}$ for $U=\left(u, v, h_{1}, \ldots, h_{N}\right)$, that is,

$$
\left\{\begin{array}{l}
\lambda u(x)-v(x)=\widetilde{u}(x), \\
\lambda v(x)-\left(a u^{\prime}(x)-b \sum_{j=1}^{N} h_{j}(x)\right)^{\prime}=\widetilde{v}(x), \\
\lambda h_{j}(x)-\left(a_{j} b_{j} u^{\prime}(x)-b_{j} h_{j}(x)\right)=\widetilde{h_{j}}(x), j=1,2, \ldots, N, \\
u(0)=u(1)=0,
\end{array}\right.
$$

to get

$$
\left\{\begin{array}{l}
v(x)=\lambda u(x)-\widetilde{u}(x), \\
h_{j}(x)=\frac{1}{\lambda+b_{j}}\left(a_{j} b_{j} u^{\prime}(x)+\widetilde{h_{j}}(x)\right), j=1,2, \ldots, N
\end{array}\right.
$$

and

$$
\left\{\begin{array}{l}
\theta^{\prime}(x)=\lambda^{2} u(x)-\lambda \widetilde{u}(x)-\widetilde{v}(x) \\
\theta(x)=p(\lambda) u^{\prime}(x)-\sum_{j=1}^{N} \frac{b}{\lambda+b_{j}} \widetilde{h}_{j}(x) \\
u(0)=u(1)=0
\end{array}\right.
$$

where $p(\lambda)$ is given by (3.22). There are two cases:

Case I: $\lambda \notin \Lambda$. In this case, $p(\lambda) \neq 0$. Since by Lemma $3.1,0 \in \rho(\mathcal{B})$, we only need consider the case of $\lambda \neq 0$. Now, we can rewrite (3.41) as the following first order system of differential equations:

$$
\left\{\begin{array}{l}
\frac{\mathrm{d}}{\mathrm{d} x}\left(\begin{array}{c}
u(x) \\
\theta(x)
\end{array}\right)=\left(\begin{array}{cc}
0 & \frac{1}{p(\lambda)} \\
\lambda^{2} & 0
\end{array}\right)\left(\begin{array}{l}
u(x) \\
\theta(x)
\end{array}\right)+\left(\begin{array}{c}
\frac{1}{p(\lambda)} V(x) \\
-\lambda \widetilde{u}(x)-\widetilde{v}(x)
\end{array}\right), \\
u(0)=u(1)=0,
\end{array}\right.
$$

where

$$
V(x)=\sum_{j=1}^{N} \frac{b}{\lambda+b_{j}} \widetilde{h_{j}}(x) .
$$

Let

$$
A(\lambda)=\left(\begin{array}{cc}
0 & \frac{1}{p(\lambda)} \\
\lambda^{2} & 0
\end{array}\right)
$$

Then

$$
\mathrm{e}^{A(\lambda) x}=\left(\begin{array}{ll}
a_{11}(\lambda, x) & a_{12}(\lambda, x) \\
a_{21}(\lambda, x) & a_{22}(\lambda, x)
\end{array}\right)
$$

where

$$
\left\{\begin{array} { l } 
{ a _ { 1 1 } ( \lambda , x ) = \operatorname { c o s h } ( \frac { \lambda } { \sqrt { p ( \lambda ) } } x ) , } \\
{ a _ { 2 1 } ( \lambda , x ) = \lambda \sqrt { p ( \lambda ) } \operatorname { s i n h } ( \frac { \lambda } { \sqrt { p ( \lambda ) } } x ) , }
\end{array} \left\{\begin{array}{l}
a_{12}(\lambda, x)=\frac{1}{\lambda \sqrt{p(\lambda)}} \sinh \left(\frac{\lambda}{\sqrt{p(\lambda)}} x\right), \\
a_{22}(\lambda, x)=\cosh \left(\frac{\lambda}{\sqrt{p(\lambda)}} x\right) .
\end{array}\right.\right.
$$


The general solution of (3.42) is given by

$$
\left(\begin{array}{l}
u(x) \\
\theta(x)
\end{array}\right)=\mathrm{e}^{A(\lambda) x}\left(\begin{array}{c}
u(0) \\
\theta(0)
\end{array}\right)-\int_{0}^{x} \mathrm{e}^{A(\lambda)(x-\gamma)}\left(\begin{array}{c}
\frac{1}{p(\lambda)} V(\gamma) \\
-\lambda \widetilde{u}(\gamma)-\widetilde{v}(\gamma)
\end{array}\right) \mathrm{d} \gamma .
$$

By $u(0)=0$, it has,

$$
u(x)=a_{12}(\lambda, x) \theta(0)-\int_{0}^{x}\left[\frac{1}{p(\lambda)} a_{11}(\lambda, x-\gamma) V(\gamma)+a_{12}(\lambda, x-\gamma)(-\lambda \widetilde{u}(\gamma)-\widetilde{v}(\gamma))\right] \mathrm{d} \gamma
$$

and

$$
\theta(x)=a_{22}(\lambda, x) \theta(0)-\int_{0}^{x}\left[\frac{1}{p(\lambda)} a_{21}(\lambda, x-\gamma) V(\gamma)+a_{22}(\lambda, x-\gamma)(-\lambda \widetilde{u}(\gamma)-\widetilde{v}(\gamma))\right] \mathrm{d} \gamma
$$

Since $\lambda \notin \sigma_{p}(\mathcal{B})$, by $(3.25)$

$$
a_{12}(\lambda, 1)=\frac{1}{\lambda \sqrt{p(\lambda)}} \sinh \left(\frac{\lambda}{\sqrt{p(\lambda)}}\right) \neq 0 .
$$

By the boundary condition $u(1)=0$, it has

$$
\theta(0)=\frac{1}{a_{12}(\lambda, 1)} \int_{0}^{1}\left[\frac{1}{p(\lambda)} a_{11}(\lambda, 1-\gamma) V(\gamma)+a_{12}(\lambda, 1-\gamma)(-\lambda \widetilde{u}(\gamma)-\widetilde{v}(\gamma))\right] \mathrm{d} \gamma
$$

Hence $u$ is uniquely determined by (3.44). By the second equation of (3.41) and (3.45), we know that $u^{\prime} \in$ $L^{2}(0,1)$. This together with $(3.40)$ shows that $(\lambda I-\mathcal{B})^{-1}$ exists and is bounded, or $\lambda \in \rho(\mathcal{B})$.

Case II: $\lambda \in \Lambda$. In this case, $\lambda \neq 0$. By (3.41),

$$
\left\{\begin{array}{l}
u(x)=\frac{1}{\lambda^{2}}\left(\lambda \widetilde{u}(x)+\widetilde{v}(x)-V^{\prime}(x)\right) \\
u(0)=u(1)=0
\end{array}\right.
$$

where $V$ is given by (3.43). Since $\widetilde{u} \in H_{0}^{1}(0,1),(3.47)$ means that (3.39) admits a solution if and only if $V$ is differentiable, and

$$
\widetilde{v}(0)-V^{\prime}(0)=\widetilde{v}(1)-V^{\prime}(1)=0 .
$$

Thus $\lambda \notin \rho(\mathcal{B})$. The result follows by combining of these two cases.

In order to investigate the residual and continuous spectrum of $\mathcal{B}$, we need the adjoint operator $\mathcal{B}^{*}$.

Lemma 3.8. Let $\mathcal{B}$ be defined by (3.6). Then

$$
\mathcal{B}^{*}\left(\begin{array}{c}
u \\
v \\
h_{1} \\
\vdots \\
h_{N}
\end{array}\right)^{\top}=\left(\begin{array}{c}
-v+\frac{1}{a} \sum_{j=1}^{N} a_{j} b_{j} \int_{0}^{x} h_{j}(\tau) \mathrm{d} \tau \\
-a u^{\prime \prime} \\
b v^{\prime}-b_{1} h_{1} \\
\vdots \\
b v^{\prime}-b_{N} h_{N}
\end{array}\right)^{\top},
$$


with

$$
D\left(\mathcal{B}^{*}\right)=\left\{\left(\begin{array}{c}
u \\
v \\
h_{1} \\
\vdots \\
h_{N}
\end{array}\right)^{\top} \mid \begin{array}{l}
u, v, \sum_{j=1}^{N} a_{j} b_{j} \int_{0}^{x} h_{j}(\tau) \mathrm{d} \tau \in H_{0}^{1}(0,1), h_{j} \in L^{2}(0,1), j=1, \ldots, N . \\
u^{\prime \prime}
\end{array}\right\} .
$$

Theorem 3.9. Let $\mathcal{B}$ be defined by (3.6). Then

$$
\sigma_{r}(\mathcal{B})=\emptyset, \quad \sigma_{c}(\mathcal{B})=\Lambda,
$$

where $\sigma_{r}(\mathcal{B})$ and $\sigma_{c}(\mathcal{B})$ denotes the set of residual and continuous spectrum of $\mathcal{B}$, respectively.

Proof. By Lemma 3.3 and Theorem 3.7, we only need to prove $\Lambda \cap \sigma_{r}(\mathcal{B})=\emptyset$. Since $\lambda \in \sigma_{r}(\mathcal{B})$ implies $\bar{\lambda} \in \sigma_{p}\left(\mathcal{B}^{*}\right)$, it suffices to show that $\Lambda \cap \sigma_{p}\left(\mathcal{B}^{*}\right)=\emptyset$. Suppose that $\mathcal{B}^{*} U=\lambda U$ for $\lambda \in \mathbb{C}$ and $0 \neq U=\left(u, v, h_{1}, \ldots, h_{N}\right) \in$ $D\left(\mathcal{B}^{*}\right)$. Then

$$
\left\{\begin{array}{l}
-v(x)+\frac{1}{a} \sum_{j=1}^{N} a_{j} b_{j} \int_{0}^{x} h_{j}(\tau) \mathrm{d} \tau=\lambda u(x), \\
-a u^{\prime \prime}(x)=\lambda v(x), \\
b v^{\prime}(x)-b_{j} h_{j}(x)=\lambda h_{j}(x), \quad j=1,2, \ldots, N, \\
v(0)=v(1)=0 .
\end{array}\right.
$$

When $\lambda \neq-b_{j}, j=1,2, \ldots, N, v$ satisfies

$$
\left\{\begin{array}{l}
\left(a-b \sum_{j=1}^{N} \frac{a_{j} b_{j}}{\lambda+b_{j}}\right) v^{\prime \prime}(x)=\lambda^{2} v(x), \\
v(0)=v(1)=0 .
\end{array}\right.
$$

For any $\lambda \in \Lambda$, it has $v=0$. This implies that $U=0$. Therefore, $\lambda \notin \sigma_{p}\left(\mathcal{B}^{*}\right)$. So, $\Lambda \cap \sigma_{p}\left(\mathcal{B}^{*}\right)=\emptyset$. The proof is complete.

\subsection{Riesz basis property}

Now, we study the Riesz basis property for system (3.3). To this purpose, we need the following Theorem 3.10, which was originally proved in [12]. Since [12] is not published, we attach its brief proof as appendix in this paper.

Theorem 3.10. Let $A$ be a densely defined closed linear operator in a Hilbert space $H$ with isolated eigenvalues $\left\{\lambda_{i}\right\}_{1}^{\infty}$ and $\sigma_{r}(A)=\emptyset$. Let $\left\{\phi_{n}\right\}_{1}^{\infty}$ be a Riesz basis for $H$. Suppose that there are $N_{0} \geq 1$ and a sequence of generalized eigenvectors $\left\{\psi_{n}\right\}_{N_{0}}^{\infty}$ of $A$ such that

$$
\sum_{n=N_{0}}^{\infty}\left\|\psi_{n}-\phi_{n}\right\|_{H}^{2}<\infty .
$$

Then there exist $M\left(\geq N_{0}\right)$ number of generalized eigenvectors $\left\{\psi_{n_{0}}\right\}_{1}^{M}$ such that $\left\{\psi_{n_{0}}\right\}_{1}^{M} \cup\left\{\psi_{n}\right\}_{M+1}^{\infty}$ forms a Riesz basis for $H$. 
Theorem 3.11. Let $\mathcal{B}$ be defined by (3.6). Then

(i) There is a sequence of generalized eigenfunctions of $\mathcal{B}$, which forms a Riesz basis for the state space $\mathcal{H}$.

(ii) All eigenvalues except finitely many are algebraically simple.

(iii) $\mathcal{B}$ generates a $C_{0}$-semigroup $\mathrm{e}^{\mathcal{B} t}$ on $\mathcal{H}$.

Therefore, for the semigroup $\mathrm{e}^{\mathcal{B} t}$, the Spectrum-determined growth condition holds: $\omega(\mathcal{B})=s(\mathcal{B})$, where $\omega(\mathcal{B})=\lim _{t \rightarrow \infty} \frac{1}{t}\left\|\mathrm{e}^{\mathcal{B} t}\right\|$ is the growth order of $\mathrm{e}^{\mathcal{B} t}$ and $s(\mathcal{B})=\sup \{\operatorname{Re} \lambda \mid \lambda \in \sigma(\mathcal{B})\}$ is the spectral bound of $\mathcal{B}$.

Proof. Since from Theorem 3.6, all eigenvalues are located in some left half complex plane, the other parts follow directly from (i) and (ii). So we only need to prove (i) and (ii). For any $n \in \mathbb{N}^{+}$, set

$$
\begin{gathered}
V_{n 0}=\left(\frac{1}{n \pi} \sin n \pi x, i \sqrt{a} \sin n \pi x, 0, \ldots, 0\right) \\
\left\{\begin{array}{c}
\varphi_{n 0}=(\sqrt{a} \cos n \pi x, i \sqrt{a} \sin n \pi x, 0, \ldots, 0)+(0,1,1, \ldots, 1) \mathcal{O}\left(n^{-1}\right), \\
\varphi_{n k}=\left(\sqrt{a}, 0, \frac{a_{1} b_{1}}{\lambda_{n k}+b_{1}}, \ldots, \frac{a_{N} b_{N}}{\lambda_{n k}+b_{N}}\right) \cos n \pi x+(0,1,1, \ldots, 1) \mathcal{O}\left(n^{-1}\right), \\
k=1,2, \ldots, N .
\end{array}\right.
\end{gathered}
$$

Define the reference sequence:

$$
\left\{\begin{array}{l}
\psi_{n 0}=(\sqrt{a} \cos n \pi x, i \sqrt{a} \sin n \pi x, 0, \ldots, 0), \\
\psi_{n k}=\left(0,0, \frac{a_{1} b_{1}}{\lambda_{n k}+b_{1}}, \ldots, \frac{a_{N} b_{N}}{\lambda_{n k}+b_{N}}\right) \cos n \pi x, k=1,2, \ldots, N .
\end{array}\right.
$$

Since $b_{j} \neq b_{k}, \lambda_{n j} \neq \lambda_{n k}, 1 \leq j<k \leq N$, a direct computation shows that

$$
\operatorname{det}\left(\begin{array}{cccc}
\frac{a_{1} b_{1}}{\lambda_{n 1}+b_{1}} & \frac{a_{1} b_{1}}{\lambda_{n 2}+b_{1}} & \cdots & \frac{a_{1} b_{1}}{\lambda_{n N}+b_{1}} \\
\frac{a_{2} b_{2}}{\lambda_{n 1}+b_{2}} & \frac{a_{2} b_{2}}{\lambda_{n 2}+b_{2}} & \cdots & \frac{a_{2} b_{2}}{\lambda_{n N}+b_{2}} \\
\cdots & \cdots & \cdots & \cdots \\
\frac{a_{N} b_{N}}{\lambda_{n 1}+b_{N}} & \frac{a_{N} b_{N}}{\lambda_{n 2}+b_{N}} & \cdots & \frac{a_{N} b_{N}}{\lambda_{n N}+b_{N}}
\end{array}\right) \neq 0
$$

Hence,

$$
\left\{\psi_{n 0}, \overline{\psi_{n 0}}, \psi_{n 1}, \psi_{n 2}, \ldots, \psi_{n N}\right\}_{1}^{\infty}
$$

forms a Riesz basis for $\mathcal{H}_{1}=\left(L^{2}(0,1)\right)^{N+2}$. By (3.54), (3.55) and Theorem 3.6, there exists an $N_{0} \in \mathbb{N}^{+}$, such that,

$$
\begin{aligned}
& \sum_{n=N_{0}}^{\infty}\left[\left\|U_{n 0}-V_{n 0}\right\|_{\mathcal{H}}^{2}+\left\|\overline{U_{n 0}}-\overline{V_{n 0}}\right\|_{\mathcal{H}}^{2}+\sum_{k=1}^{N}\left\|U_{n k}-\frac{U_{n 0}+\overline{U_{n 0}}}{2}-\psi_{n k}\right\|_{\mathcal{H}}^{2}\right] \\
& =\sum_{n=N_{0}}^{\infty}\left[\left\|\varphi_{n 0}-\psi_{n 0}\right\|_{\mathcal{H}_{1}}^{2}+\left\|\overline{\varphi_{n 0}}-\overline{\psi_{n 0}}\right\|_{\mathcal{H}_{1}}^{2}+\sum_{k=1}^{N}\left\|\varphi_{n k}-\frac{\varphi_{n 0}+\overline{\varphi_{n 0}}}{2}-\psi_{n k}\right\|_{\mathcal{H}_{1}}^{2}\right] \\
& <\infty .
\end{aligned}
$$

By Theorem 3.10, (i) and hence (ii) hold true. The proof is complete.

Combing Theorems 3.6, 3.7 and 3.11, we conclude the exponential stability of system (3.3). 
Theorem 3.12. System (3.3) is exponentially stable, that is,

$$
E(t) \leq M e^{-\omega t} E(0),
$$

for some $M, \omega>0$, where $E(t)$ is given by (3.4).

\section{Appendix A. Proof of Theorem 3.10}

Let $\operatorname{sp}(A)$ denote the root subspace of $A$ that is a closed linear span of all generalized eigenfunctions of $A$. Let $E\left(\lambda_{i}, A\right)$ denote the projection on the space of generalized eigenvectors of $A$ corresponding to $\lambda_{i}$, that is, the subspace spanned by all those $\phi_{i}$ satisfying $\left(\lambda_{i}-A\right)^{n} \phi_{i}=0$ for some positive integer $n$. We have following lemmas.

Lemma A.1. Let $A$ be a linear operator in a Hilbert space $H$ with isolated eigenvalues and residual spectrum $\left\{\lambda_{i}\right\}_{1}^{\infty}, \rho(A) \neq \emptyset$. Let

$$
\sigma_{\infty}=\left\{x \mid E\left(\lambda_{i}, A\right) x=0, i \geq 1\right\} .
$$

Then $\sigma_{\infty}$ is either 0 or infinite dimensional.

Proof. Suppose first that $A$ is bounded and $0<\operatorname{dim} \sigma_{\infty}<\infty$. Since $\sigma_{\infty}$ is invariant subspace of $A$, that is, $A \sigma_{\infty} \subset \sigma_{\infty}, A$ has at least one eigenvector $x_{\infty} \in \sigma_{\infty}$ such that $A x_{\infty}=\eta x_{\infty}$ for some constant $\eta$. So $\eta=\lambda_{i}$ for some $i$, and hence,

$$
x_{\infty}=E\left(\lambda_{i}, A\right) x_{\infty}=0,
$$

which is a contradiction. So (A.1) holds true.

If $A$ is unbounded. Take $\lambda_{0} \in \rho(A)$ such that $\left|\lambda_{0}-\lambda_{i}\right| \geq \varepsilon>0$ for all $i \geq 1$. Let $T=\left(\lambda_{0}-A\right)^{-1}$, $\mu_{i}=\left(\lambda_{0}-\lambda_{i}\right)^{-1}, i=1,2, \ldots$ Then it is well-known that

$$
\lambda_{i} \in \sigma_{p}(A) \text { if and only if } \mu_{i} \in \sigma_{p}(T), \lambda_{i} \in \sigma_{r}(A) \text { if and only if } \mu_{i} \in \sigma_{r}(T)
$$

and

$$
E\left(\lambda_{i}, A\right)=E\left(\mu_{i}, T\right), \text { for all } i \geq 1
$$

Hence

$$
\sigma_{\infty}=\left\{x \mid E\left(\mu_{i}, T\right) x=0, \mu_{i} \in \sigma_{p}(T) \cup \sigma_{r}(T)\right\} .
$$

Since $T$ is bounded, $\sigma_{\infty}$ is either 0 or infinite dimensional.

Lemma A.2. Let $A$ be a densely defined closed operator in a Hilbert space $H$ with isolated eigenvalues $\left\{\lambda_{i}\right\}_{1}^{\infty}$. Then

$$
H=\operatorname{sp}(A) \oplus \sigma_{\infty}^{*},
$$

where

$$
\sigma_{\infty}^{*}=\left\{x \mid E\left(\bar{\lambda}_{i}, A^{*}\right) x=0, \lambda_{i} \in \sigma_{p}(A)\right\} .
$$

Proof. By a well-known fact $\sigma\left(A^{*}\right)=\{\bar{\lambda} \mid \lambda \in \sigma(A)\}, \bar{\lambda}_{i}$ is an isolated spectral point of $A^{*}$ and so $E\left(\bar{\lambda}_{i}, A^{*}\right)$ makes sense. For any $f \in E\left(\lambda_{i}, A\right) H, g^{*} \in \sigma_{\infty}^{*}$, we have $E\left(\lambda_{i}, A\right) f=f$. And hence

$$
\left\langle f, g^{*}\right\rangle=\left\langle E\left(\lambda_{i}, A\right) f, g^{*}\right\rangle=\left\langle f, E\left(\bar{\lambda}_{i}, A^{*}\right) g^{*}\right\rangle=0 .
$$

So $\operatorname{sp}(A) \subset\left(\sigma_{\infty}^{*}\right)^{\perp}$. If $f \notin \operatorname{sp}(A)$, then there exists a functional $g^{*}$ such that

$$
\left\langle f, g^{*}\right\rangle=1,\left\langle h, g^{*}\right\rangle=0, \text { for all } h \in \operatorname{sp}(A) .
$$


For any $w \in H$, it follows from $E\left(\lambda_{i}, A\right) w \in \operatorname{sp}(A)$ that

$$
\left\langle w, E\left(\bar{\lambda}_{i}, A^{*}\right) g^{*}\right\rangle=\left\langle E\left(\lambda_{i}, A\right) w, g^{*}\right\rangle=0 .
$$

By the arbitrary of $w$, it has $E\left(\bar{\lambda}_{i}, A^{*}\right) g^{*}=0$. That is $g^{*} \in \sigma_{\infty}^{*}$. Hence $f \notin\left(\sigma_{\infty}^{*}\right)^{\perp}$. Therefore, $\operatorname{sp}(A)=\left(\sigma_{\infty}^{*}\right)^{\perp}$, proving (A.2).

The next Lemma A.3 comes from [19].

Lemma A.3. Let $\left\{\phi_{n}\right\}_{1}^{\infty}$ be a Riesz basis for a Hilbert space H. Suppose there are $N_{0} \geq 1$ and an $\omega$-linearly independent sequence $\left\{\psi_{n}\right\}_{N_{0}}^{\infty}$ such that

$$
\sum_{n=N_{0}}^{\infty}\left\|\psi_{n}-\phi_{n}\right\|^{2}<\infty .
$$

Then $\left\{\psi_{n}\right\}_{N_{0}}^{\infty}$ forms a Riesz basis for the subspace spanned by itself.

Proof of Theorem 3.10. Condition (3.52) implies that there exists an $M \geq N_{0}$ such that $\left\{\phi_{n}\right\}_{1}^{M} \cup\left\{\psi_{n}\right\}_{M+1}^{\infty}$ forms a Riesz basis for $H$. In particular, $(\operatorname{sp}(A))^{\perp}$ is finite dimensional. This together with (A.2) shows that $\sigma_{\infty}^{*}$ is finite dimensional. It is known that $\bar{\lambda} \in \sigma_{p}\left(A^{*}\right) \cup \sigma_{r}\left(A^{*}\right)$ if and only if $\lambda \in \sigma_{p}(A) \cup \sigma_{r}(A)$. By our assumption, $\sigma_{p}\left(A^{*}\right) \cup \sigma_{r}\left(A^{*}\right)=\left\{\bar{\lambda}_{i}\right\}_{1}^{\infty}$. By Lemma A1, it follows that $\sigma_{\infty}^{*}=\{0\}$. Therefore,

$$
\operatorname{sp}(A)=H .
$$

Suppose that $\left\{\psi_{\alpha}\right\} \cup\left\{\psi_{n}\right\}_{M}^{\infty}$ is the "maximal" $\omega$-linearly independent set of generalized eigenvector of $A$, that is, $\left\{\psi_{\alpha}\right\} \cup\left\{\psi_{n}\right\}_{M}^{\infty}$ is an $\omega$-linearly independent set and if adding another extra generalized eigenvector of $A$ to $\left\{\psi_{\alpha}\right\} \cup\left\{\psi_{n}\right\}_{M}^{\infty}$, the extended set is not $\omega$-linearly independent anymore. By Lemma A.3, $\left\{\psi_{\alpha}\right\} \cup\left\{\psi_{n}\right\}_{M}^{\infty}$ forms a Riesz basis for the subspace spanned by itself, which is the whole space as we just proved.

Since a proper subset of a Riesz basis can not be a Riesz basis, it follows from condition (3.52) and Bari's theorem (see Sect. 2 of [9] in p. 309) that the number of $\left\{\psi_{\alpha}\right\}$ is just $M$. The proof is complete.

\section{REFERENCES}

[1] G. Amendola, M. Fabrizio, J.M. Golden and B. Lazzari, Free energies and asymptotic behaviour for incompressible viscoelastic fluids. Appl. Anal. 88 (2009) 789-805.

[2] H.T. Banks, G.A. Pinter, L.K. Potter, B.C. Munoz and L.C. Yanyo, Estimation and control related issues in smart material structures and fluids, The 4th International Conference on Optimization: Techniques and Applications. Perth, Australia (1998) $19-34$.

[3] H.T. Banks, J.B. Hood and N.G. Medhin, A molecular based model for polymer viscoelasticity: intra- and inter-molecular variability. Appl. Math. Model. 32 (2008) 2753-2767.

[4] G. Chen, S.A. Fulling, F.J. Narcowich and S. Sun, Exponential decay of energy of evolution equations with locally distributed damping. SIAM J. Control Optim. 51 (1991) 266-301.

[5] S.P. Chen, K.S. Liu and Z.Y. Liu, Spectrum and stability for elastic systems with global or local Kelvin-Voigt damping. SIAM J. Appl. Math. 59 (1999) 651-668.

[6] C.M. Dafermos, An abstract Volterra equation with application to linear viscoelasticity. J. Differential Equations 7 (1970) $554-569$.

[7] M. Fabrizio and B. Lazzari, On the existence and asymptotic stability of solutions for linearly viscoelastic solids. Arch. Ration. Mech. Anal. 116 (1991) 139-152.

[8] M. Fabrizio and S. Polidoro, Asymptotic decay for some diferential systems with fading memory. Appl. Anal. 81 (2002) $1245-1264$.

[9] I.C. Gohberg and M.G. Krein, Introduction to the Theory of Linear Nonselfadjoint Operators, Trans. Math. Monographs 18. AMS Providence (1969).

[10] B.Z. Guo, Riesz basis approach to the stabilization of a flexible beam with a tip mass, SIAM J. Control Optim. 39 (2001) 1736-1747.

[11] B.Z. Guo, Riesz basis property and exponential stability of controlled Euler-Bernoulli beam equations with variable coefficients. SIAM J. Control Optim. 40 (2002) 1905-1923.

[12] B.Z. Guo and H. Zwart, Riesz Spectral System. Preprint, University of Twenty, the Netherlands (2001). 
[13] B.Z. Guo, J.M. Wang and G.D. Zhang, Spectral analysis of a wave equation with Kelvin-Voigt damping. Z. Angew. Math. Mech. 90 (2010) 323-342.

[14] B. Jacob, C. Trunk and M. Winklmeier, Analyticity and Riesz basis property of semigroups associated to damped vibrations. J. Evol. Equ. 8 (2008) 263-281.

[15] K.S. Liu and Z.Y. Liu, On the type of $C_{0}$-semigroup associated with the abstract linear viscoelastic system. Z. Angew. Math. Phys. 47 (1996) 1-15.

[16] K.S. Liu and Z.Y. Liu, Exponential decay of energy of the Euler-Bernoulli beam with locally distributed Kelvin-Voigt damping. SIAM J. Control Optim. 36 (1998) 1086-1098.

[17] K.S. Liu and Z.Y. Liu, Exponential decay of energy of vibrating strings with local viscoelasticity. Z. Angew. Math. Phys. 53 (2002) 265-280.

[18] Y.Z. Liu and S.M. Zheng, On the exponential stability of linear viscoelasticity and thermoviscoelasticity. Quart. Appl. Math. 54 (1996) 21-31.

[19] B.P. Rao, Optimal energy decay rate in a damped Rayleigh beam, Contemporary Mathematics. RI, Providence 209 (1997) $221-229$.

[20] M. Renardy, On localized Kelvin-Voigt damping. Z. Angew. Math. Mech. 84 (2004) 280-283.

[21] J.E.M. Rivera and H.P. Oquendo, The transmission problem of viscoelastic waves. Acta Appl. Math. 62 (2000) 1-21.

[22] H.S. Tzou and J.H. Ding, Optimal control of precision paraboloidal shell structronic systems. J. Sound Vib. 276 (2004) 273-291.

[23] J.M. Wang, B.Z. Guo and M.Y. Fu, Dynamic behavior of a heat equation with memory. Math. Methods Appl. Sci. 32 (2009) $1287-1310$.

[24] H.L. Zhao, K.S. Liu and Z.Y. Liu, A note on the exponential decay of energy of a Euler-Bernoulli beam with local viscoelasticity. J. Elasticity $\mathbf{7 4}$ (2004) 175-183.

[25] H.L. Zhao, K.S. Liu and C.G. Zhang, Stability for the Timoshenko beam system with local Kelvin-Voigt damping. Acta Math. Sinica (Engl. Ser.) 21 (2005) 655-666.

[26] E. Zuazua, Exponential decay for the semilinear wave equation with locally distributed damping. Comm. Partial Differential Equations 15 (1990) 205-235. 